\title{
Multiple Synchronous Tumours: A Peculiar Clinical Case
}

\author{
Maria Leitão, Tiago Alpoim, Manuela Machado* \\ Medical Oncology Department, IPO, Porto, Portugal \\ Email: *m.machado.fn@gmail.com
}

How to cite this paper: Leitão, M., Alpoim, T. and Machado, M. (2019) Multiple Synchronous Tumours: A Peculiar Clinical Case. Journal of Cancer Therapy, 10, 602-608. https://doi.org/10.4236/jct.2019.107049

Received: June 14, 2019

Accepted: July 21, 2019

Published: July 24, 2019

Copyright $\odot 2019$ by author(s) and Scientific Research Publishing Inc. This work is licensed under the Creative Commons Attribution International License (CC BY 4.0).

http://creativecommons.org/licenses/by/4.0/

\section{c) (i) Open Access}

\begin{abstract}
Background: Due to improvements in diagnosis, the better outcomes of oncological patients and the increase in the average age, the incidence of synchronous tumours is likely to increase. Aim: To reflect on the challenges of a case with multiple integrated diagnostic and therapeutic approaches and to bring to consideration the increasing prevalence of similar situations. Case Presentation: In this clinical case, the authors describe the evolution of an asymptomatic patient with several synchronous tumours (a GEJ primary adenocarcinoma, a low grade urothelial carcinoma in situ, a localized squamous cell lung carcinoma and 2 IPMNs). Conclusion: The challenge of this case lies in the difficult diagnostic approach, the assembly of a multidisciplinary and time-sensitive treatment plan and the individualized follow-up, due to lack of guidelines. More research is needed in this area.
\end{abstract}

\section{Keywords}

Gastric Cancer, Multiple Primary Neoplasms, Synchronous

\section{Introduction}

The evolution of Medicine has encompassed new screening methods (including population-based screening procedures) and new imaging techniques that have made possible the diagnosis of tumours in earlier, localized stages. Moreover, the advances in oncological treatments have increased the overall survival across an array of neoplasms, including in advanced disease. Consequently, the incidence of synchronous tumours (by definition, primary tumours with different histologies) is thought to be on the rise. [1].

The challenge resides in coordinating the treatment strategy in order to ensure optimal action of systemic therapies on different diseases, with favorable timings and the least possible combined toxicity. 
On the other hand, the possibility of synchronous tumours on radiology exams being interpreted as metastasis of a single primary neoplasm has great implications on treatment choices and prognosis, raising the issue of when to biopsy masses found during the staging process.

\section{Case Report}

A.H.M.P., born in 1952, was a smoker for over 20 years, suffered from type 2 diabetes mellitus, hypertension and high cholesterol. He had no family history of cancer.

In 2017, at 64 years old, the patient was diagnosed with a tumour of the gastric-esophageal junction (GEJ) at $36 \mathrm{~cm}$-classified as Siewert II, on a routine upper endoscopy. The biopsy revealed a tubular adenocarcinoma and an in situ carcinoma. An ultra-sound endoscopy was performed, staging the tumour as uT2N0. No relevant alterations were found in the broncofibroscopy, such as in the colonoscopy.

A thoraco-abdominopelvic CT scan was requested, which reported additionally a lesion on the bladder wall and pancreatic cysts. The patient was submitted to a transurethral bladder resection of a $2 \mathrm{~cm}$ neoplasia in the right ureterovesical junction and a $0.5 \mathrm{~cm}$ neoplasia on the right side bladder wall. Mytomicin C was instilled post-operatively. The pathology report of the specimens identified a low grade urothelial in situ carcinoma.

A 8F-fluorodeoxyglucose positron emission tomography (PET-FDG) showed avid glucose uptake on a right pulmonary nodule, in addition to the gastro-esophageal and vesical masses (Figures 1-3). The CT-guided lung biopsy found malignant cells, suggestive of carcinoma, positive for p63 and CK7, negative for CK20 and TTF-1, with possible origin in a squamous cell lung carcinoma

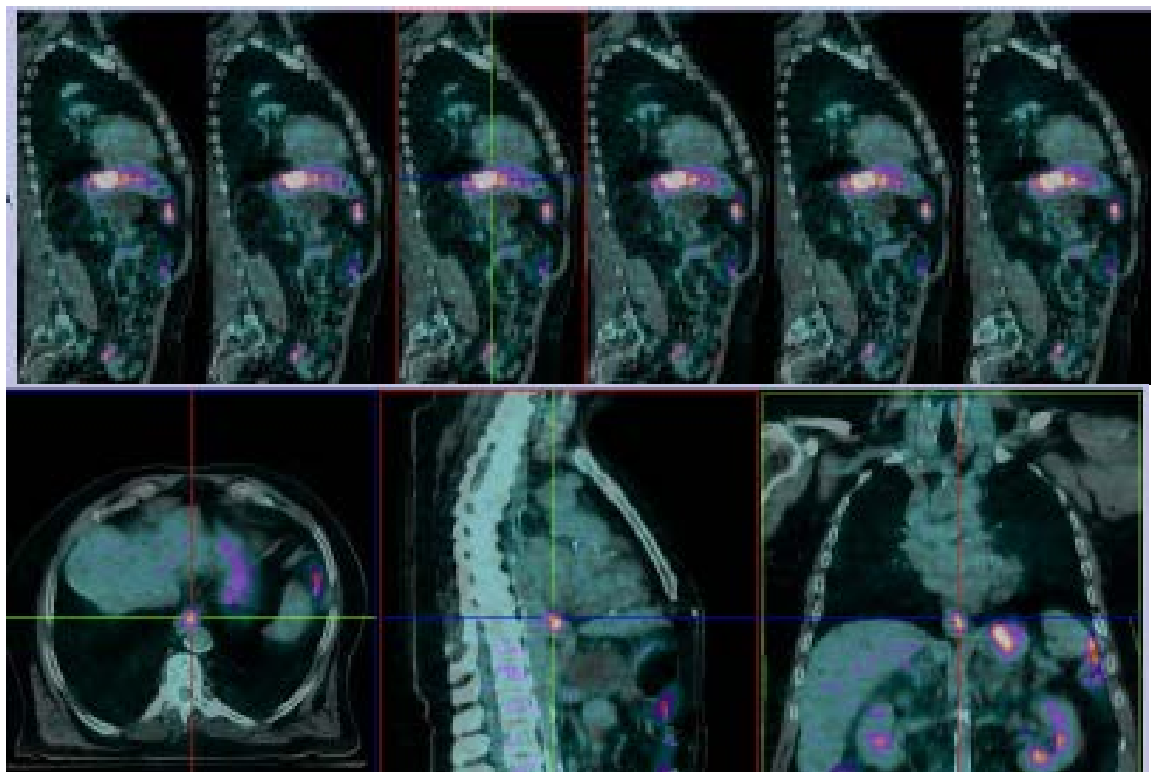

Figure 1. PET-FDG with glucose hypercaptation in the distal esophagus and cardia, extending to the lesser gastric curvature. 


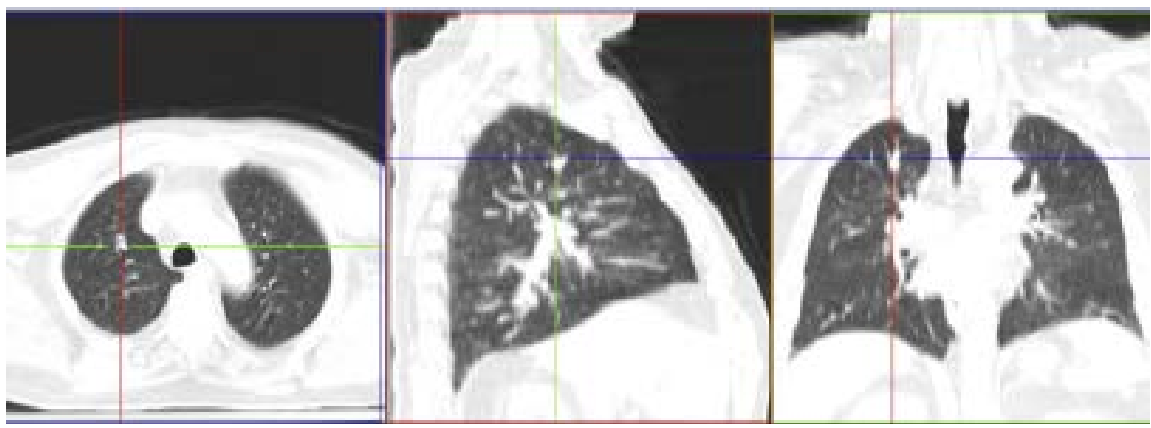

Figure 2. PET-CT scan showing pulmonary lesion.

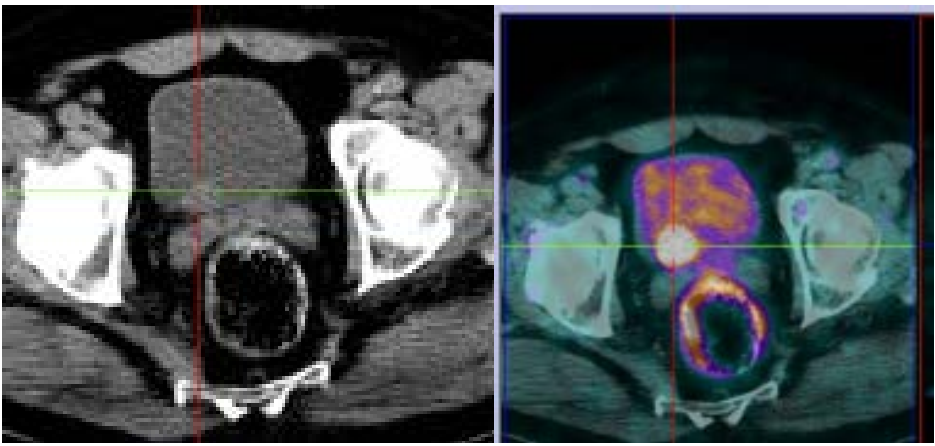

Figure 3. PET-CT scan with bladder wall mass.

or an urothelial carcinoma. Considering the non-invasive nature of the urothelial primary carcinoma discovered, the nodule was regarded as synchronous lung cancer, cT1aN0M0.

The case was discussed by a multidisciplinary team. It was decided to perform neoadjuvant chemoradiotherapy (CRT) to the GEJ tumour and curative-intent SBRT to the lung, followed by surgical intervention to the GEJ lesion. The urothelial in situ carcinoma had been completely removed, therefore observation was proposed.

The patient exhibited general tolerance to the CRT, which was performed with 5 cycles of carboplatin and paclitaxel (carboplatin AUC dosing of 2) and radiation with $41.4 \mathrm{~Gy}$ in 23 fractions, 5 days a week, according to the VMAT technique. SBRT to the pulmonary lesion was carried out in 3 fractions of $15 \mathrm{~Gy}$ (total 45 Gy), every 72 hours, with 3 DRT technique and respiratory gating. Finally, a transhiatal subtotal esophagogastrectomy with pancreatic cystectomy was performed. The JEG primary tumour was then classified as ypT3N3aM0 R0. The two pancreatic lesions excised were analyzed and the pathological findings were consistent with intrapancreatic mucinous neoplasias (IPMNs) with high-grade dysplasia, without invasion foci, probable branch-duct type.

The patient was then without evidence of disease and therefore was kept in tight observation, with trimestral oncology visits with history and physical exams and follow-up imaging exams every 3 months.

In January 2019, a pleural effusion was identified on CT scan. Due to suspicion of recurrence, a PET CT was requested, revealing FDG hypermetabolism in 
the esophagus and esophageal anastomosis of uncertain etiology, bone metastasis in L3 vertebra (Figure 4) and suspected liver metastization (Figure 5). Upper endoscopy showed polipoid lesions involving the esophageal mucous layer circumferentially, with adenocarcinoma involvement on histology. The cytology of the pleural effusion, as well as the esophageal, bone and liver biopsies, were consistent with esophagogastric origin, HER-2 negative. The tumour expresses MLH1, PMS2, MSH2 and MSH6 normally, therefore lacking microsatellite instability. Epstein-Barr virus (EBV) was negative.

The patient remained asymptomatic, with an ECOG performance status of 0 . Physical examination was unremarkable with the exception of peripheral oedema, due to CTCAE Grade 2 hypoalbuminemia.

Chemotherapy was initiated with mFOLFOX6. Treatment with zoledronic acid was also started. Disease evaluation through CT scan will be performed soon.

The patient is still in observation by the specialty of Urology due to the bladder primary, with regular cystoscopies, the last of which was performed in April and showed no signs of recurrence.

A specialized genetics consultation was requested, but their feedback on the case was not yet released.

The patient has expressed his consent to the publication of this case.

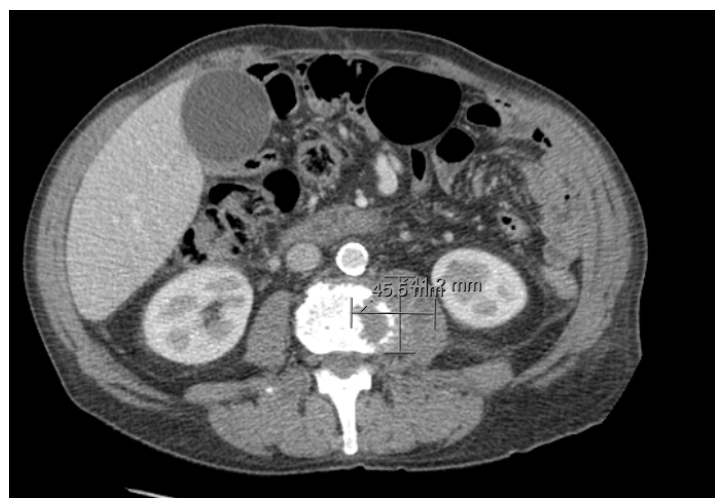

Figure 4. Vertebral bone metastasis on CT scan.

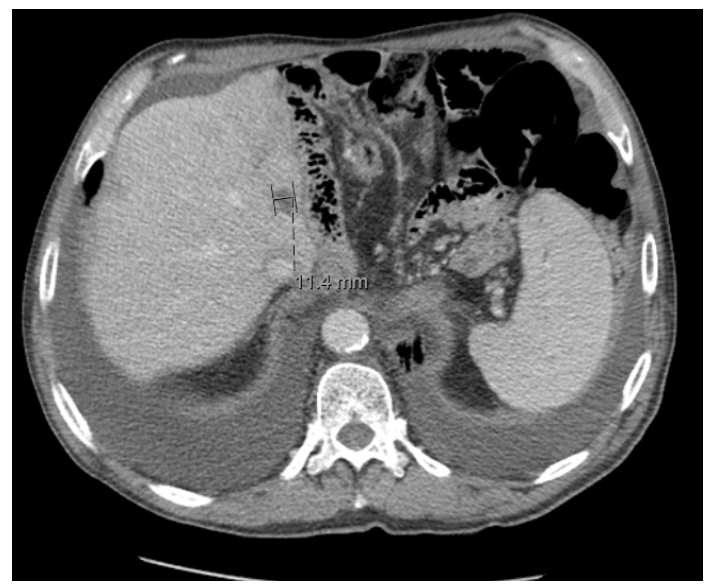

Figure 5. Hepatic metastasis on CT scan. 


\section{Discussion}

The definition of synchronous primary tumours implies neoplasms with different histologies that occur simultaneously or within 6 months of one another (otherwise, the classification would be of metachronous tumours) [2].

In this clinical case, we identify an asymptomatic patient with a synchronous GEJ primary adenocarcinoma, a low grade urothelial carcinoma in situ, a localized squamous cell lung carcinoma and 2 IPMNs. With this rare cluster of findings, mostly in early stages of disease, this case becomes an uncommon and thought-provoking clue of our still insufficient understanding of the origins of cancer.

Several questions may arise about the management of this patient, including the initial treatment order, the choice of neoadjuvant versus peri-operatory chemotherapy, the need for multiple biopsies throughout the disease course and how to perform follow up.

The primary treatment plan was devised with the natural history of the different cancers and the timings for the different technical timings in mind: the urothelial carcinoma would be more easily cured and thus, became the starting point of this therapeutic course. The localized pulmonary disease was treated with SBRT, which was feasible during the waiting period before surgery. Consequently, it was possible to minimize the time lapses without therapy.

Even though the gastric tumour is classified as Siewert II, it was pondered whether FLOT or CROSS regimens would be the best treatment option. In our institution, Siewert II tumours are usually treated with CROSS regimen and the two drugs of the scheme also are frequently are used in lung cancer, hence it seemed to be the best option [3] [4] [5].

The question arises on the need for several biopsies and when to perform them. In the initial approach, biopsies of the bladder wall thickening were requested because it constitutes an uncommon site of gastric metastization. Even though $20 \%$ of gastric cancers eventually develop lung metastasis, the lung lesion was biopsied due to the imagiological features that suggested the possibility of primary etiology. In the recurrent setting, multiple biopsies were needed to clarify which of the tumours had relapsed, considering that both lung and gastric cancers may originate bone metastasis. In the CROSS trial, progression free survival was $71 \%$ at 1 year and $60 \%$ at 2 years [1]. Given that SBRT shows local control rates of $90 \%$ at 5 years [6], it seemed less likely that the recurrence would have pulmonary origin, but it remained a possibility with crutial repercussions on management and prognosis.

Some clinical features may point towards the diagnosis of multiple primary tumours vs metastasis of a single primary tumour and as a result should prompt other biopsies, including: atypical locations or timing of metastatic spread in the context of the supposed primary tumour, higher tumour burden than would be expected comparatively to the tumour marker load, exposure to know carcinogenic substances or imagiological characteristics [1]. 
There are no guidelines or studies on the follow up of patients with multiple synchronous tumours. The observation plan was individualized to reflect the needs of the patient.

In 2014, the Cancer Genome Atlas research network published the classification of 4 subtypes of gastric cancer, based on their molecular signature: Epstein Barr virus positive, tumours microsatellite unstable (MSI) tumours, genomically stable (GS) tumours and chromosomal instability (CIN) tumours. Of these, EBV positive and MSI subtypes have a higher mutational burden and this fact may play a role on prognosis and treatment: EBV positive tumours tend to have better prognosis and MSI tend to be better candidates to immunotherapy in later treatment lines. Our patient's recurrence was not positive for MSI or EBV [7].

As first line chemotherapy the option was mFOLFOX6. Although not being consensual the association of a platinum and a fluoropyrimidine is the most used scheme.

\section{Conclusion}

The authors decided to publish this case because of the challenging decision making process in a situation that is expected to become more frequent, albeit still largely unknown. More research is needed in this area to provide answers on what are the best options for these patients.

\section{Conflicts of Interest}

The authors declare no conflicts of interest regarding the publication of this paper.

\section{References}

[1] Vogt, A., et al. (2017) Multiple Primary Tumours: Challenges and Approaches: A Review. ESMO Open, 2, e000172. https://doi.org/10.1136/esmoopen-2017-000172

[2] Testori, A., et al. (2015) Multiple Primary Synchronous Malignant Tumors. BMC Research Notes, 8, 730. https://doi.org/10.1186/s13104-015-1724-5

[3] Shapiro, J., et al. (2015) Neoadjuvant Chemoradiotherapy plus Surgery versus Surgery Alone for Oesophageal or Junctional Cancer (CROSS): Long-Term Results of a Randomised Controlled Trial. The Lancet Oncology, 16, 1090-1098. https://doi.org/10.1016/S1470-2045(15)00040-6

[4] Al Batran, S.E., et al. (2019) Perioperative Chemotherapy with Fluorouracil plus Leucovorin, Oxaliplatin, and Docetaxel versus Fluorouracil or Capecitabine plus Cisplatin and Epirubicin for Locally Advanced, Resectable Gastric or Gastro-Oesophageal Junction Adenocarcinoma (FLOT4): A Randomised, Phase 2/3 Trial. The Lancet, 393, 1948-1957. https://doi.org/10.1016/S0140-6736(18)32557-1

[5] Machado, M., Magalhães, H. and Sousa, M. (2018) Perioperative Treatment in Gastric Cancer-Developments in Patient Selection. Journal of Cancer Therapy, 9 , 101-105. https://doi.org/10.4236/jct.2018.92011

[6] Postmus, P.E., Kerr, K.M., Oudkerk, M., Senan, S., Waller, D.A., Vansteenkiste, J., Escriu, C. and Peters, S. (2017) Early-Stage and Locally Advanced (Non-Metastatic) Non-Small-Cell Lung Cancer: ESMO Clinical Practice Guidelines. Annals of On- 
cology, 28, iv1-iv21. https://doi.org/10.1093/annonc/mdx222

[7] Bass, A.J., et al. (2014) Comprehensive Molecular Characterization of Gastric Adenocarcinoma. Nature, 513, 202-209. https://doi.org/10.1038/nature13480 